\title{
Morphological study of the sensory units innervating cat salivary ducts
}

\author{
Tetsu Yamamoto, Satoshi Yamazaki and Sanya Sakada \\ Department of Physiology (Ghief: Prof. Sanya Sakada), Tokyo Dental College, \\ 1-2-2, Masago, Chiba City, Chiba 260, Japan
}

[Accepted for publication: March 16, 1983]

Key words: innervation / sensory units / sensory nerve endings / salivary ducts

The existence of baroceptors in the submandibular gland and its duct wall has been proven by electrophysiological technique ${ }^{1)}$. However, no morphological observation has been made of the innervation of salivary ducts. This paper reports on the appearances of sensory units innervating the cat salivary ducts.

\section{Materials and Methods}

Four cats weighing $1.0 \sim 3.3 \mathrm{~kg}$ were used. Under anesthesia with sodium pentobarbital, they were dissected to take out the full length of the bilateral sublingual, the submandibular and the parotid ducts. The fresh tissues were then stained immediately with a $0.01 \%$ methylene blue solution for $15 \sim 20$ minutes at $37^{\circ} \mathrm{C}$, fixed in a $8 \%$ ammonium molybdate solution for 12 hours at $4{ }^{\circ} \mathrm{C}$ and washed in running water for 30 minutes. The specimens were then spread on glass slides and mounted wholly in a $50 \%$ glycerin solution for investigation with a light microscope.

\section{Results}

Many encapsulated corpuscles in addition to free nerve endings were observed on the walls of the sublingual and the submandibular ducts in parts close to the oral mucosa while there were no organized sensory nerve endings on the wall of parotid ducts. These encapsulated corpuscles with an inner core and capsule were varied in shape and size. Almost all of them $(91.7 \%)$, as shown in Fig. 1, had a non-branched axon terminal in an elongated capsule, $16 \sim 38 \mu \mathrm{m}$ in diam- eter and $40 \sim 252 \mu \mathrm{m}$ in length, and the fiber diameter was $3.5 \sim 6.0 \mu \mathrm{m}$ at the distal internodal segments. The other types of encapsulated corpuscles were those having a branched axon $(7.7 \%)$ and those having a divided capsule $(0.6 \%)$.

The encapsulated corpuscles and the free nerve endings constituted the sensory units separately. Single sublingual and submandibular ducts taken out from a young cat (weighing $1.5 \mathrm{~kg}$ ) were innervated by 36 and 18 encapsulated corpuscle units respectively, each linking $1 \sim 43$ encapsulated corpuscles in the parent fiber (Fig. 2). Consequently, these sublingual and submandibular ducts

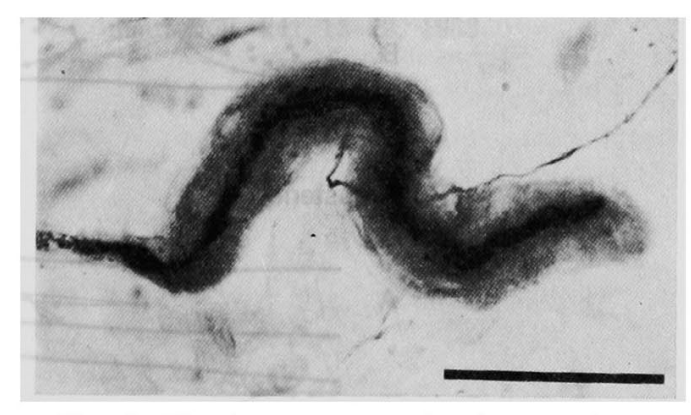

Fig. 1 Simple type encapsulated corpuscle on the wall of sublingual duct. Methylene blue staining, scale: $50 \mu \mathrm{m}$.

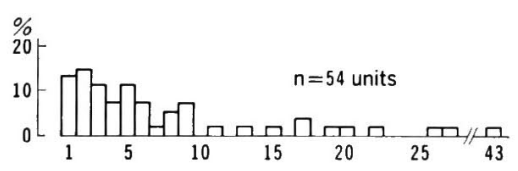

Fig. 2 The number of encapsulated corpuscles per single unit. 


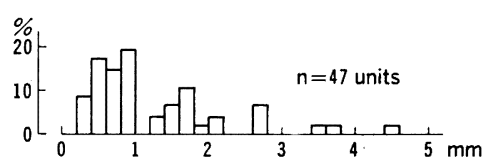

Fig. 3 The major axes of receptory fields of the units composed of two or more encapsulated corpuscles.

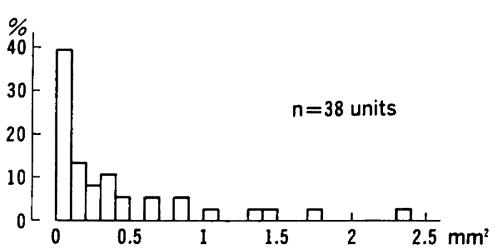

Fig. 4 The area of receptory fields of the units composed of three or more encapsulated corpuscles.

\section{Anterior parts}

A

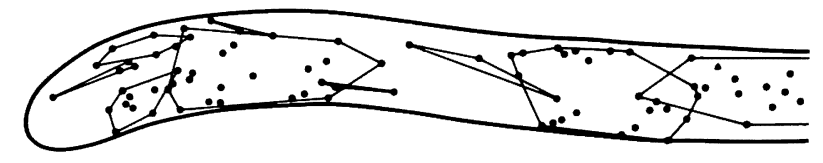

B

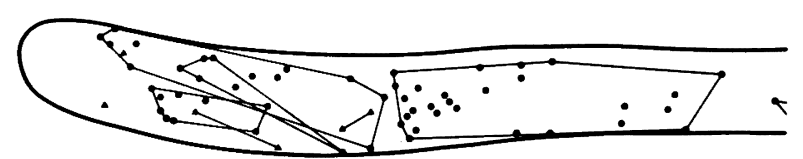

Central parts

A

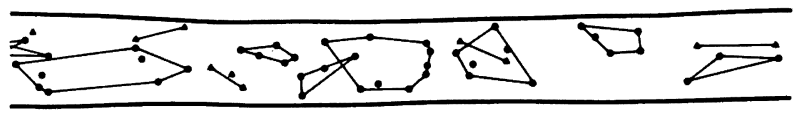

B

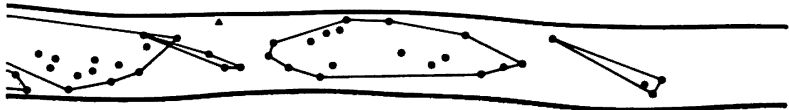

\section{Posterior parts}

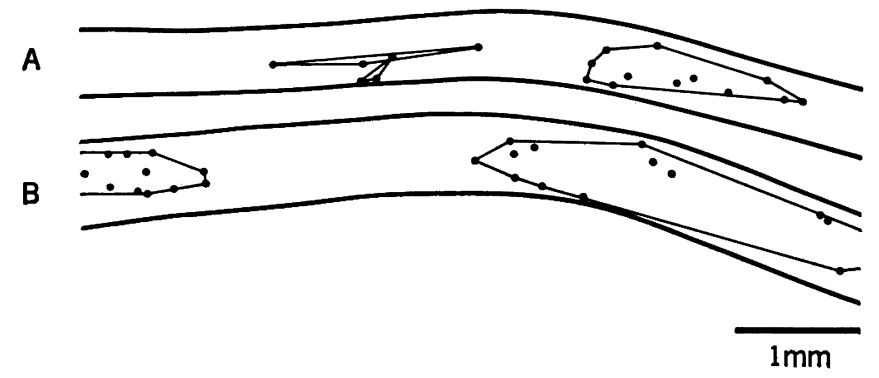

Fig. 5 Distribution of the sensory units composed of one or two encapsulated corpuscles ( $\mathbf{\Delta}$ ) and those composed of three or more encapsulated corpuscles $(\bullet)$.

The receptory fields of each units are shown by enclosed lines.

A: Sublingual ducts, B: Submandibular ducts. 
had 208 and 200 encapsulated corpuscles respectively.

The major axes of the receptory fields of the units composed of two or more encapsulated corpuscles were $0.27 \sim 4.59 \mathrm{~mm}$ (Fig. 3 ) and the areas of those composed of three or more encapsulated corpuscles were $0.01 \sim$ $2.38 \mathrm{~mm}^{2}$ (Fig. 4). The distribution density of the encapsulated corpuscle units and their nerve terminals were higher in the vicinity of the orifice than in the posterior parts of the ducts (Fig. 5).

\section{Discussion}

The encapsulated corpuscles observed in this study were similar to those in the alveo$\operatorname{lar}^{2,3)}$ and the lip mucosa ${ }^{4)}$ in the cat and the lip mucosa in the mouse ${ }^{5}$. The number of encapsulated corpuscles per single unit and the area of receptory fields of the units were almost the same as those in the cat alveolar mucosa ${ }^{2)}$.

These specialized nerve terminations distributed in the wall of sublingual and submandibular ducts can be regarded as mechanoreceptors based on their morphological characteristics. It is however inadequate to consider that these mechanoreceptors respond to the changes of the salivary flow in the ducts so as to control salivation, because the encapsulated corpuscles are lacking on the parotid duct wall. It is suggested that these encapsulated corpuscles are related to the secretion of reflex saliva to receive external stimulation caused by lingual or food movements.

\section{References}

1) Kawamura, Y. and Funakoshi, M.: A study on baro-receptive nerve fibers from the submandibular gland of the dog. Jpn. J. Physiol. 14: 528-537, 1964

2) Sakada, S., Ichikawa, R., Ohono, H. and Tsukamoto, Y.: The nerve terminals of a single sensory unit in the cat's oral mucosa. Medicine and Biology 100: 157-162, 1980. (in Japanese)

3) Sakada, S.: Electrophysiological and histological properties of rapidly and slowly adapting mechanoreceptors in cat's oral mucosa, Jaw Position and Jaw Movement (Kubota, K., Nakamura, Y. and Schumacher, G.H. eds.), p. 150-160, VEB Verlag Volk und Gesundheit, Berlin, 1980.

4) Malinovský, L.: The variability of encapsulated corpuscles in the upper lip and tongue of the domestic cat (Felis ocreata L., $f$. domestica). Folia Morphol. 14: 175-191, 1966.

5) Yamamoto, T. and Sakada, S.: Morphology and distribution of the sensory nerve endings in the mouse labial mucosa. Bull. Tokyo dent. Coll. 24: 13-22, 1983. 